\title{
Imaging G-Ratio in Multiple Sclerosis Using High-Gradient Diffusion MRI and Macromolecular Tissue Volume
}

\author{
(D) F. Yu, (D) . Fan, (D) Q. Tian, (DC. Ngamsombat, (D) N. Machado, (D).D. Bireley, (D)A.W. Russo, (D) A. Nummenmaa, (D)T. Witzel,
} (D)L.L. Wald, (DE.C. Klawiter, and (DS.Y. Huang

\begin{abstract}
BACKGROUND AND PURPOSE: Remyelination represents an area of great therapeutic interest in multiple sclerosis but currently lacks a robust imaging marker. The purpose of this study was to use high-gradient diffusion MRI and macromolecular tissue volume imaging to obtain estimates of axonal volume fraction, myelin volume fraction, and the imaging g-ratio in patients with MS and healthy controls and to explore their relationship to neurologic disability in MS.
\end{abstract}

MATERIALS AND METHODS: Thirty individuals with MS (23 relapsing-remitting MS, 7 progressive MS) and 19 age-matched healthy controls were scanned on a 3T MRI scanner equipped with $300 \mathrm{mT} / \mathrm{m}$ maximum gradient strength using a comprehensive multishell diffusion MRI protocol. Macromolecular tissue volume imaging was performed to quantify the myelin volume fraction. Diffusion data were fitted to a 3-compartment model of white matter using a spheric mean approach to yield estimates of axonal volume fraction. The imaging g-ratio was calculated from the ratio of myelin volume fraction and axonal volume fraction. Imaging metrics were compared between groups using 2-sided $t$ tests with a Bonferroni correction.

RESULTS: The mean g-ratio was significantly elevated in lesions compared with normal-appearing WM $(0.74$ vs $0.67, P<.001)$. Axonal volume fraction $(0.17$ vs $0.23, P<.001)$ and myelin volume fraction $(0.17$ vs $0.25, P<.001)$ were significantly lower in lesions than normal-appearing WM. Myelin volume fraction was lower in normal-appearing WM compared with that in healthy controls ( 0.25 vs $0.27, P=.009)$. Disability, as measured by the Expanded Disability Status Scale, was significantly associated with myelin volume fraction $(\beta=-40.5, P=.001)$ and axonal volume fraction $(\beta=-41.0, P=.016)$ in normal-appearing WM.

CONCLUSIONS: The imaging g-ratio may serve as a biomarker for the relative degree of axonal and myelin loss in MS.

ABBREVIATIONS: AVF = axonal volume fraction; EDSS = Expanded Disability Status Scale; $\mathrm{HC}=$ healthy controls; MSFC = multiple sclerosis functional composite; MTV = macromolecular tissue volume; MVF = myelin volume fraction; NAWM = normal-appearing white matter; NODDI = neurite orientation dispersion and density imaging; PMS = progressive MS; qMT = quantitative magnetization transfer; RRMS = relapsing-remitting MS

M ultiple sclerosis is a disease of the CNS characterized by inflammatory demyelination and axonal loss. Demyelination predisposes axons to immune-mediated injury, resulting in axonal loss that is thought to be the substrate of permanent disability. ${ }^{1}$ Myelin repair occurs to a variable extent, and promoting remyelination represents an area of great therapeutic interest. ${ }^{2,3}$ Unfortunately, conventional MRI is unable to distinguish remyelination from myelin loss and axonal damage. This lack of

Received July 27, 2019; accepted after revision August 12.

From the Division of Neuroradiology (F.Y.), Department of Radiology, University of Texas Southwestern Medical Center, Dallas, Texas; Athinoula A. Martinos Center for Biomedical Imaging (Q.F., Q.T., C.N., A.N., T.W., L.L.W., S.Y.H.), Department of Radiology, Massachusetts General Hospital, Charlestown, Massachusetts; Department of Neurology (N.M., J.D.B., A.W.R., E.C.K., S.Y.H.) and Division of Neuroradiology (S.Y.H.), Department of Radiology, Massachusetts General Hospital, Boston, Massachusetts; and Harvard-MIT Division of Health Sciences and Technology (L.L.W., S.Y.H.), Massachusetts Institute of Technology, Cambridge, Massachusetts. sensitivity and specificity highlights the need for more specific imaging approaches.

Research reported in this publication was supported by the National Institute of Neurological Disorders and Stroke of the National Institutes of Health under award Nos. K23NS096056 (S.Y.H.) and K23NS078044 (E.C.K.), by the National Institute of Biomedical Imaging and Bioengineering of the National Institutes of Health under award No. ROOEBO15445 (A.N.), the National Multiple Sclerosis Society (PR-1412-02194 to E.C.K.), the Radiological Society of North America (F.Y., S.Y.H.), the Conrad N. Hilton Foundation (S.Y.H.), the Altschul Foundation (E.C.K.), and an American Heart Association Postdoctoral Fellowship (Q.F.).

Paper previously presented, in part, at: Annual Meeting of the American Society of Neuroradiology, April 22-27, 2017; Long Beach, California; and Annual Meeting of the Eastern Neuroradiological Society, August 24-27, 2017; Toronto, Ontario, Canada, where it was awarded the Norman E. Leeds Award for Best Scientific Paper.

Please address correspondence to Fang Frank Yu, MD, UT Southwestern Medical Center, 5323 Harry Hines Blvd, Dallas, TX 75390; e-mail: Frank.yu@ utsouthwestern.edu; @FrankFYuMD1

\footnotetext{
- Indicates open access to non-subscribers at www.ajnr.org

Indicates article with supplemental on-line table.

http://dx.doi.org/10.3174/ajnr.A6283
} 


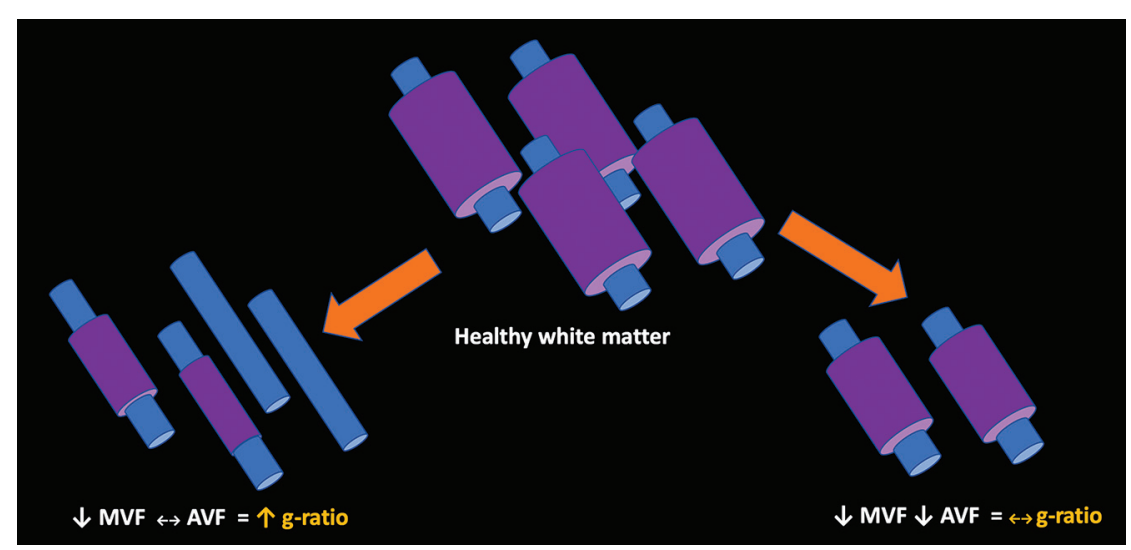

FIG 1. Schematic diagram outlining different possible outcomes for the g-ratio based on alterations in myelination and axonal integrity relative to healthy white matter. Left, When demyelination predominates and axonal integrity is preserved, the g-ratio is elevated. Right, When axonal loss occurs with concordant loss of myelin, the g-ratio may be relatively conserved.

Advanced diffusion MRI methods such as diffusional kurtosis imaging, ${ }^{4}$ neurite orientation dispersion and density imaging (NODDI), ${ }^{5}$ and AxCaliber, ${ }^{6}$ provide metrics such as the axonal volume fraction (AVF) that may be more specific to axonal microstructure than DTI metrics in white matter disease ${ }^{7}$ and neurodegeneration. ${ }^{8}$ The availability of higher gradient strengths on clinical and research MRI scanners also offers improved sensitivity to intra-axonal water diffusion compared with measurements at conventional gradient strengths. ${ }^{9-11}$ Quantitative MRI markers sensitive to myelin volume fraction (MVF) include quantitative magnetization transfer (qMT), ${ }^{12}$ myelin water imaging, ${ }^{13}$ and macromolecular tissue volume (MTV) imaging. ${ }^{14}$

Combining myelin and axonal metrics has the potential to offer a richer in vivo characterization of white matter integrity than either metric alone. The synergistic combination of MVF and AVF can be used to estimate an aggregate myelin g-ratio, which is defined as the ratio of the inner-to-outer diameter of the myelin sheath. ${ }^{15}$ The g-ratio could be used to extract the degree of axonal myelination, illuminating disease evolution that might otherwise be challenging to interpret. For example, lesions with demyelination as the predominant pathology would demonstrate an increased g-ratio (Fig 1). On the other hand, in lesions with concomitant myelin and axonal loss, the g-ratio would remain unchanged.

Several studies have used MR imaging to interrogate the g-ratio in the brain ${ }^{15-18}$ and spinal cord, ${ }^{19,20}$ referred to here as the imaging g-ratio. Previous imaging studies in MS demonstrated an increase in g-ratio within lesions compared with the normalappearing white matter (NAWM) using NODDI for estimation of AVF, and qMT and synthetic MRI for estimation of MVF, respectively. ${ }^{15,17}$ We postulate that the use of high-gradient diffusion MRI imaging should provide consistent estimates of AVF compared with those reported in previous studies and, when combined with measurements of MVF, should yield consistent trends in the imaging g-ratio between lesions and NAWM.

The purpose of this study was to use high-gradient diffusion MRI and MTV imaging to obtain estimates of AVF, MVF, and imaging g-ratio in patients with MS and healthy controls (HC) and to explore the relationship of these imaging markers with neurologic disability in MS. We hypothesized that lesions would show evidence of demyelination and axonal loss manifesting as decreased MVF, decreased AVF, and increased gratio relative to NAWM and white matter of HC.

\section{MATERIALS AND METHODS}

This prospective study was approved by the Massachusetts General Hospital institutional review board and is compliant with the Health Insurance Portability and Accountability Act guidelines. All participants provided written informed consent.

\section{Subjects}

Thirty patients with a clinical diagnosis of MS and 19 age-matched healthy volunteers were prospectively recruited from March 2016 through March 2018 (On-line Table). Of the 30 patients with MS, 23 had relapsing-remitting MS (RRMS), while 7 had progressive MS (PMS; consisting of 2 primary-progressive and 5 secondary-progressive MS patients. Inclusion criteria for subjects with MS were a diagnosis of clinically definite MS, absence of clinical relapse within 3 months, and being on stable disease-modifying treatment or no treatment for at least 6 months. Exclusion criteria for all subjects were evidence of other structural brain diseases, severe claustrophobia, or other contraindications to MR imaging.

Neurologic disability in the patients with MS was assessed using the Expanded Disability Status Scale (EDSS) and Multiple Sclerosis Functional Composite (MSFC). A boardcertified neurologist blinded to the imaging results conducted a standard clinical examination, which was used in the calculation of the EDSS. A trained examiner administered the MSFC3 , and the $z$ score was calculated using the Symbol Digit Modalities Test, Timed 25-Foot Walk, and Timed Nine-Hole Peg Test.

\section{MRI Acquisition}

All subjects were imaged on a 3T MRI scanner (Magnetom Connectom; Siemens, Erlangen, Germany) with a maximum gradient strength of $300 \mathrm{mT} / \mathrm{m}$ and a maximum slew rate of $200 \mathrm{~T} /$ $\mathrm{m} / \mathrm{s}$. A custom-built 64 -channel phased array head coil was used for signal reception. ${ }^{21}$ Diffusion data were acquired using a diffusion-weighted spin-echo single-shot EPI sequence in the sagittal plane with 2-mm isotropic voxel size, TE/TR $=77 / 3600 \mathrm{~ms}$, parallel imaging acceleration factor $\mathrm{R}=2$, simultaneous multislice imaging with a slice acceleration factor of 2 , and anterior-to-posterior phase encoding. Interspersed $b=0$ images were acquired every 16 images. A multishell diffusion imaging protocol was performed using a diffusion gradient pulse duration of $8 \mathrm{~ms}$, diffusion times of 19 and $49 \mathrm{~ms}$, and 8 diffusion gradient increments linearly spaced from 30 to $290 \mathrm{mT} / \mathrm{m}$ per diffusion time, for a total of $16 \mathrm{~b}$-values. The diffusion gradients were applied in 32 directions for $\mathrm{b}$-values of $<2300 \mathrm{~s} / \mathrm{mm}^{2}$ and 64 directions for $\mathrm{b}$ - 
values of $>2300 \mathrm{~s} / \mathrm{mm}^{2}$, uniformly distributed on a sphere. The maximum b-value was $17,800 \mathrm{~s} / \mathrm{mm}^{2}$. A set of $5 b=0$ images with a reversed-phase encoding direction was acquired to correct for susceptibility-induced distortions. The diffusion MRI protocol was designed to include the minimum number of diffusion-weighted imaging volumes to obtain reproducible estimates of apparent axon diameter and density compared with a more extensive acquisition, ${ }^{22}$ while keeping the scan time within an acceptable duration for imaging patients ( $<1$ hour). The total acquisition time was 51 minutes.

MTV data for myelin quantification were acquired using a multiple flip angle spoiled gradient-echo $3 \mathrm{D}-\mathrm{FLASH}$ sequence at 1 - $\mathrm{mm}$ isotropic resolution with $\mathrm{TE} / \mathrm{TR}=2.74 / 20 \mathrm{~ms}$ and flip angle $=4^{\circ}, 10^{\circ}, 20^{\circ}$. The duration of each 3D-FLASH sequence was 5 minutes 6 seconds, resulting in a total acquisition time of 15 minutes 18 seconds. Structural images were acquired including a T1-weighted multiecho MPRAGE sequence at 1-mm isotropic resolution with $\mathrm{TE} / \mathrm{TR}=1.15,3.03,4.89,6.75 \mathrm{~ms} / 2530 \mathrm{~ms}$, $\mathrm{TI}=1100 \mathrm{~ms}, \mathrm{R}=3$, and flip angle $=7^{\circ}$ (acquisition time of 3 minutes 58 seconds), and a $3 \mathrm{D}$-FLAIR sequence at $0.9-\mathrm{mm}$ isotropic resolution with $\mathrm{TE} / \mathrm{TR} / \mathrm{TI}=389 / 5000 / 1800 \mathrm{~ms}$ and $\mathrm{R}=2$ (acquisition time of 5 minutes 47 seconds).

\section{Data Processing and Analysis}

Diffusion Imaging. All data were corrected for gradient nonlinearity using in-house software. ${ }^{23}$ Susceptibility- and eddy current-induced distortions and motion in the diffusion-weighted images were corrected using topup (https://fsl.fmrib.ox.ac.uk/fsl/ fslwiki/topup) and eddy (https://fsl.fmrib.ox.ac.uk/fsl/fslwiki/ eddy) in FSL (https://fsl.fmrib.ox.ac.uk). ${ }^{24-26}$

A recently developed approach for inferring axonal compartment size and volume fraction from a multicompartment spherical mean signal model of white matter was used to quantify the axonal volume fraction. ${ }^{27}$ A 3-compartment model of restricted diffusion within impermeable cylindric axons, hindered Gaussian diffusion in the extra-axonal space, and free diffusion in CSF was used to model water diffusion in white matter. The spherically averaged form of the signal model was fitted using Markov chain Monte Carlo sampling to yield orientation-independent estimates of restricted volume fraction $\left(f_{r}\right)$, hindered diffusion coefficient, and CSF volume fraction $\left(\mathrm{f}_{\mathrm{csf}}\right) .^{28}$ The longitudinal diffusion coefficient was assumed to be $1.7 \times 10^{-3} \mathrm{~mm}^{2} / \mathrm{s}$ within axons, and the free diffusivity coefficient was assumed to be $3 \mathrm{~mm}^{2} / \mathrm{s}$ (diffusivity of free water at $37^{\circ} \mathrm{C}$ ).

Macromolecular Tissue Volume. The 3D-FLASH magnitude images were corrected for $\mathrm{B}_{1}$ inhomogeneity. ${ }^{20}$ The FLASH images with flip angle $=4^{\circ}, 10^{\circ}$ were then registered to the FLASH image with flip angle $=20^{\circ}$ using the FSL Linear Registration Tool (FLIRT; http://www.fmrib.ox.ac.uk/fsl/fslwiki/ FLIRT). The equilibrium magnetization $\mathrm{M}_{0}$ (product of the coil reception profile and proton density) and $\mathrm{T} 1$ values were estimated on a voxel-wise basis. ${ }^{20}$ The macromolecular tissue volume was computed as ${ }^{14}$

1)

$$
M T V=1-\left(\frac{\text { Proton Density }}{\text { Proton Density of Free Water }}\right) .
$$

\section{Registration and Segmentation}

Cortical surface reconstruction and volumetric segmentation were performed on T1-weighted images using FreeSurfer (Version 5.3.0; http://surfer.nmr.mgh.harvard.edu). ${ }^{29,30}$ The FLASH images at flip angle $=20^{\circ}$ and $b=0$ diffusion images were coregistered to each subject's T1-weighted image using the boundary-based registration tool in FreeSurfer with $6 d f$. The resultant transformations were applied to the MTV, $\mathrm{f}_{\mathrm{r}}$, and $\mathrm{f}_{\text {csf }}$ maps for alignment with the T1weighted images.

Binary lesion masks were generated from the T1 MPRAGE and 3D-FLAIR images using an in-house automated segmentation procedure developed within the FreeSurfer image analysis suite, described in detail in Lindemer et $\mathrm{al}^{31}$ and adapted for use in patients with MS by the authors of that work. In brief, this segmentation tool performs intensity normalization of a subject's T1- and FLAIR-weighted images using a multimodal atlas and segments white matter lesions from NAWM using a multimodal Gaussian classifier array as well as individual-based heuristics. The lesion masks generated by the automated tool tended to overestimate the lesion size and were manually edited by 2 experienced neuroradiologists, who overlaid the lesion masks generated by automated segmentation on the 3D-FLAIR images and removed voxels that did not clearly contain T2/FLAIR hyperintensity in the perilesional white matter. In cases in which there were incongruences between the lesion masks generated by the 2 raters, the decisions on which voxels to include in the lesion mask were made by consensus. Whole-brain cerebral white matter masks were created by combining the FreeSurfer segmentations of right and left hemispheric white matter and the corpus callosum. Masks of NAWM in the patients with MS were generated by subtracting the lesion masks from the cerebral white matter masks.

Comparisons to prior clinical MR imaging were performed for the subjects with MS. Of these, 22 (16 RRMS) subjects had clinical contrast-enhanced MRI of the brain completed within one year before the study scan.

\section{G-Ratio Calculation}

The g-ratio-weighted maps were generated assuming that MTV reflects the MVF. ${ }^{20}$ The AVF was calculated by combining $\mathrm{f}_{\mathrm{r}}, \mathrm{f}_{\mathrm{csf}}$, and MTV: ${ }^{15,20}$

2)

$$
A V F=(1-M T V) \times\left(1-f_{c f f}\right) \times f_{r} .
$$

The g-ratio (g) was then computed as ${ }^{15}$

3)

$$
g=\sqrt{\frac{1}{1+\frac{M T V}{A V F}}} .
$$

\section{Statistical Analysis}

Statistical analysis was performed in STATA 12.1 (StataCorp, College Station, Texas). Comparisons of MVF, AVF, and the g-ratio among lesions, NAWM, and white matter in HC (HCWM) were performed using a 2-sided $t$ test, following the 1-sample Kolmogorov-Smirnov test for normality. The Mann-Whitney $U$ test was used to compare subsets of patients with MS (RRMS versus PMS). Bonferroni correction was performed to account for multiple comparisons (uncorrected $P<.05$, corrected $P<.0167$ ) 


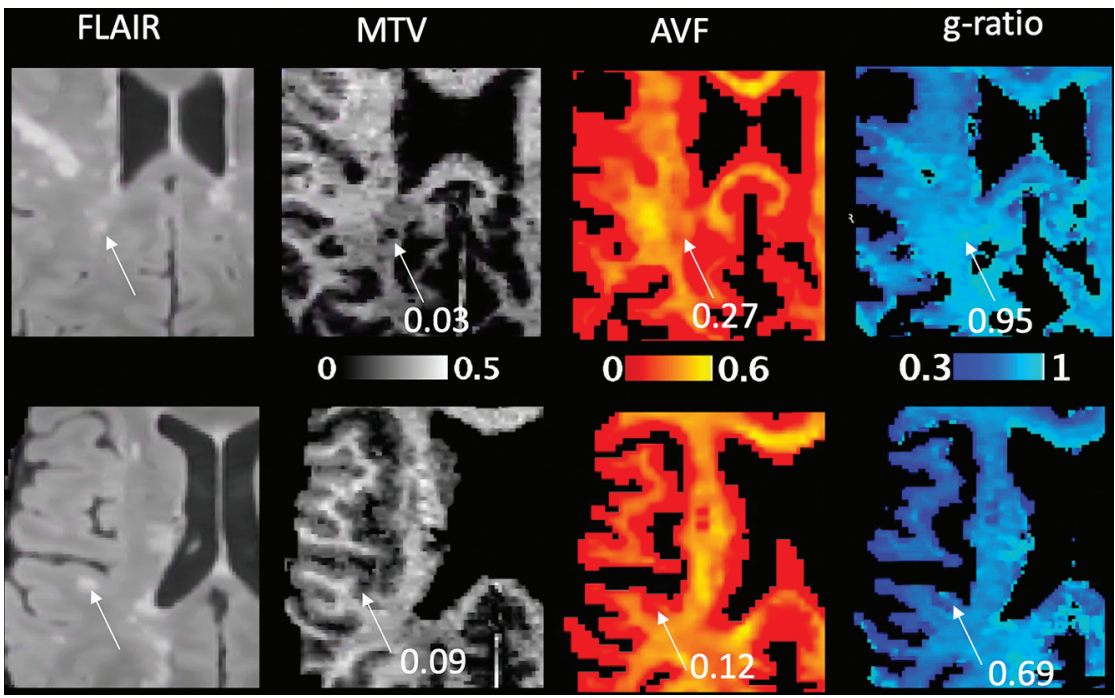

FIG 2. Representative axial T2-FLAIR image and maps of macromolecular tissue volume reflecting myelin content, axonal volume fraction, and g-ratio in a patient with RRMS. The upper row shows a lesion with an elevated g-ratio (0.95), indicating a greater degree of demyelination relative to axonal loss. The lower row shows a lesion with concordant axonal and myelin loss, which is reflected by a g-ratio similar to that of NAWM (0.69). For comparison, the conventional T2-FLAIR images do not demonstrate a discernible difference in contrast between the 2 lesions.

for each set of tissue contrasts. Raw uncorrected $P$ values surviving Bonferroni correction are reported throughout this work.

Correlations between disability scores and imaging metrics averaged in each subject were assessed using linear regression, adjusting for age and sex.

\section{RESULTS}

Demographic and clinical characteristics of the MS and HC groups are shown in the On-line Table. The MS group did not differ significantly from the HC group in age or sex ratio. Furthermore, the RRMS subgroup did not differ significantly from the combined PMS subgroups in age, sex, disease duration, or use of disease-modifying therapy. Among all subjects with MS, there were 1418 supratentorial lesions. The mean number of lesions per subject was $48.9 \pm 51$ (range, 4-212). Based on comparison with clinical scans, 10 subjects with MS had lesions (total of 17 lesions) that developed within 1 year before the study scan.

Figure 2 highlights the variability in the g-ratio as exemplified in a patient with RRMS, including a lesion with an elevated g-ratio reflecting myelin loss but preserved AVF, in comparison with a lesion with a g-ratio similar to that of NAWM. This example demonstrates the potential of the imaging g-ratio to distinguish lesions affected predominantly by demyelination from those with concordant axonal and myelin loss.

Among all patients with MS, lesion AVF and MVF were significantly lower than in NAWM, $(0.17$ versus 0.23 for AVF, $P<$ .001 , and 0.17 versus 0.25 for MVF, $P<.001$ ) (Table 1 ). The mean g-ratio was significantly elevated in lesions compared with NAWM (0.74 versus $0.67, P<.001)$. The MVF was lower in
NAWM compared with HCWM (0.25 versus $0.27, P<.009$ ). No significant difference was observed in the g-ratio and AVF of NAWM and HCWM.

When we compared imaging metrics in RRMS and PMS subgroups (Table 2), the average lesion AVF (0.14 versus $0.18, P<.006)$ and MVF $(0.13$ versus $0.18, P<.001)$ were significantly lower in PMS. The average lesion g-ratio was not significantly different between RRMS and PMS. NAWM MVF was significantly lower in PMS compared with RRMS (0.22 versus $0.26, P<.01$ ). No significant difference in AVF or g-ratio was observed for NAWM in subjects with RRMS and PMS.

Disability, measured by the EDSS, was significantly associated with MVF $(\beta=-40.5, P<.001)$ and $\operatorname{AVF}(\beta=-$ 41.0, $P<.02)$ in NAWM, with lower MVF and AVF observed with higher EDSS scores. No significant relationship was observed between the EDSS and the g-ratio in NAWM averaged in each subject $(\beta=16.5, P=.20)$. Higher EDSS scores were significantly associated with lower MVF $(\beta=-21.9$, $P=.03)$ and higher g-ratios $(\beta=11.9, P=.04)$ in lesions. Disability, measured by the MSFC-3, was significantly associated with MVF in NAWM $(\beta=45.9, P=.001)$, with lower MVF observed with lower MSFC-3 $z$ scores. Lower AVF ( $\beta=$ $35.8, P=.06)$ and a higher g-ratio in NAWM $(\beta=-25.6, P=$ $.07)$ were associated with worse performance on the MSFC-3, though the correlations did not meet statistical significance. Lower MSFC-3 $z$-scores correlated with lower MVF $(\beta=$ 31.4, $P=.003)$ and higher g-ratios $(\beta=-18.5, P=.003)$ in lesions.

\section{DISCUSSION}

In this study, we estimated AVF using a spheric mean analysis of high-gradient diffusion MRI data and quantified myelin content using MTV imaging. We examined the imaging metrics of AVF and MVF separately and combined them to probe alterations in the imaging g-ratio within lesions and NAWM in patients with MS. We found evidence of myelin and axonal loss within lesions compared with NAWM, as well as myelin loss in NAWM compared with HCWM. Lower AVF and MVF in NAWM were significant predictors of neurologic disability as measured by the EDSS and the MSFC- 3 in the case of MVF. A trend toward a higher g-ratio was observed in patients who performed worse on the EDSS and MSFC-3, though the correlations did not meet statistical significance.

MVF and AVF were significantly lower in lesions relative to NAWM, consistent with prior g-ratio imaging studies that reported both myelin and axonal loss within focal lesions in MS. ${ }^{15,17}$ The finding of an elevated g-ratio in lesions compared with NAWM suggested that myelin loss was the predominant 
Table 1: Comparison of imaging metrics in patients with multiple sclerosis and healthy controls

\begin{tabular}{lccccc}
\hline & HC & NAWM & Lesions & HC vs NAWM & NAWM vs Lesions \\
$(\boldsymbol{P}$ Value $)$ & $.15^{\mathrm{a}}$ & $(\boldsymbol{P}$ Value $)$ \\
\hline AVF & $0.24 \pm 0.02$ & $0.23 \pm 0.02$ & $0.17 \pm 0.04$ & $.009^{\mathrm{b}, \mathrm{c}}$ & $<.001^{\mathrm{a}, \mathrm{b}}$ \\
MVF & $0.27 \pm 0.02$ & $0.25 \pm 0.03$ & $0.17 \pm 0.04$ & $.17^{\mathrm{a}}$ & $<.001^{\mathrm{a}, \mathrm{b}}$ \\
G-ratio & $0.66 \pm 0.02$ & $0.67 \pm 0.03$ & $0.74 \pm 0.06$ & $<.001^{\mathrm{b}, \mathrm{d}}$ \\
\hline
\end{tabular}

a Student $t$ test.

${ }^{\mathrm{b}}$ Significance following Bonferroni correction.

${ }^{c}$ Mann-Whitney $U$ test.

${ }^{\mathrm{d}}$ Wilcoxon signed rank test.

pathology and the resulting myelin sheaths were thinner. Examining the relative change in MVF and AVF as estimated through these advanced MRI metrics may help to distinguish among acute demyelinating, remyelinating, and chronic lesions, which is not possible with conventional T2/FLAIR sequences. ${ }^{32}$ Following the initial demyelinating event, MVF would be expected to decrease while AVF would be relatively preserved, resulting in an elevation of the g-ratio. The g-ratio would be expected to normalize with more complete remyelination. Alternatively, chronic lesions with complete loss of the axolemma would also be expected to demonstrate g-ratio values approaching those of NAWM.

Lesions in patients with MS demonstrated significantly lower MVF and AVF compared with those in patients witb RRMS, consistent with more profound myelin and axonal loss. The g-ratio did not differ significantly, indicating a similar degree of relative lesion demyelination in PMS and RRMS. The NAWM of patients with PMS also showed lower MVF and AVF compared with patients with RRMS, but to a lesser degree than in lesions.

The estimated MVF and AVF were lower in NAWM compared with HCWM, though not to the same degree as in lesions. The g-ratio in NAWM also did not differ significantly from HCWM. One possible explanation is that there may be relatively concordant myelin and axonal loss in NAWM. This hypothesis is supported by previous studies of clinically progressive MS that showed that the underlying pathology shifts from one of inflammatory attacks to neurodegeneration of the NAWM. ${ }^{34}$

In evaluating the correlation between neurologic disability and MVF, AVF, and g-ratio in the NAWM, we found significant negative correlations between EDSS and MVF and AVF, indicating that clinical disability worsens as NAWM loses myelin and axonal integrity. The trend toward an elevated g-ratio with more profound disability suggests that more pronounced relative demyelination, or incomplete remyelination, may contribute to greater clinical disability.

To date, most in vivo studies of g-ratios within the CNS have been performed using NODDI to estimate $\mathrm{AVF}^{16,20,22,34}$ and $\mathrm{qMT}^{15,16,19,20,34}$ to estimate MVF. Our estimates for the mean gratio within lesions and NAWM were similar to those previously reported using qMT and NODDI. ${ }^{15}$ Hagiwara et $\mathrm{al}^{17}$ found higher lesion g-ratios, likely due to differences in their approach to myelin quantification using synthetic MRI. Specifically, their estimates for MVF were considerably lower within lesions than prior qMT estimates ${ }^{35}$ as well as our MTV results, possibly due to not including a partial volume pool to account for magnetization transfer effects, which could result in a downward bias in the MVF estimates. ${ }^{17}$ Accordingly, the estimated g-ratio in lesions was also higher.

Our estimates for AVF were generally lower than those of previous studies using NODDI, a finding we attribute to differences in the assumptions of the 2 diffusion models. The NODDI approach assumes fixed and equal parallel diffusivities in the intra- and extra-axonal space, which has been shown to produce substantial overestimation of the AVF on the order of $30 \%-$ 50\%. ${ }^{5,36}$ By comparison, in our spheric mean model, we explicitly estimated the extra-axonal hindered diffusivity, constraining it to be less than the intra-axonal parallel diffusivity, as suggested by independent validation of the compartment parallel diffusivities. ${ }^{37}$ In our work, we also took advantage of the $300-\mathrm{mT} / \mathrm{m}$ maximum gradient strength accessible on the Connectom scanner. Higher gradient strengths have been shown to increase the sensitivity of diffusion MRI experiments to intra-axonal water diffusion. ${ }^{9-11}$ The accuracy of the AVF values obtained in our study is supported by systematic numeric simulations and validation experiments on a biomimetic brain phantom composed of textile hollow fibers with restricted volume fractions ranging from 0.1 to $0.7 .^{38}$ The estimates of AVF using the spheric mean approach and related techniques are in line with those obtained from histology, ${ }^{39}$ with estimates of AVF in human white matter from electron microscopy being on the order of $0.2-0.3$, which is consistent with the AVF values estimated in healthy controls and NAWM in our study.

If substantiated in larger studies, the imaging g-ratio may be used as a biomarker to aid in patient selection for disease-modifying therapy. In particular, early-phase clinical trials for remyelinating therapies have yielded inconsistent results to date, ${ }^{33}$ possibly due to suboptimal patient selection. ${ }^{32} \mathrm{MS}$ is a heterogeneous disease with specific phenotypes that may be more

Table 2: Comparison of imaging metrics in patients with relapsing-remitting and progressive multiple sclerosis

\begin{tabular}{lcccccc}
\hline & & & & RRMS vs PMS & $\begin{array}{c}\text { RRMS vs PMS } \\
\text { Lesions (P Value) }\end{array}$ & NAWM (P Value) \\
\hline AVF & $0.18 \pm 0.04$ & $0.14 \pm 0.02$ & $0.24 \pm 0.02$ & $0.22 \pm 0.02$ & $.006^{\text {a }}$ & .06 \\
MVF & $0.18 \pm 0.03$ & $0.13 \pm 0.03$ & $0.26 \pm 0.02$ & $0.22 \pm 0.03$ & $.001^{\mathrm{a}}$ & $.01^{\mathrm{a}}$ \\
G-ratio & $0.72 \pm 0.05$ & $0.78 \pm 0.07$ & $0.67 \pm 0.02$ & $0.68 \pm 0.04$ & .13 & .39 \\
\hline
\end{tabular}

${ }^{\mathrm{a}}$ Significance following Bonferroni correction. 
responsive to remyelinating therapy, which could vary within the same patient at different time points. ${ }^{40}$ The incorporation of g-ratio-weighted imaging with myelin and axonal metrics may offer additional tools to help address this issue. Specifically, patients with acute lesions who show elevated g-ratios consistent with more profound demyelination may be more likely to respond to remyelinating therapies, whereas patients with near-normal gratios reflecting more complete remyelination, or axonal loss, may be less likely to derive a benefit.

\section{CONCLUSIONS}

In this study, we imaged the g-ratio in patients with MS using AVF derived from high-gradient diffusion MRI and MVF quantified through MTV. We found evidence of disproportionately greater myelin loss resulting in elevated g-ratios within lesions compared with NAWM. NAWM showed a lesser degree of axonal and myelin loss that was relatively concordant compared with white matter in healthy controls. Clinical disability as measured by the EDSS and MSFC-3 correlated with MVF, AVF, and, to a lesser degree, with the g-ratio in patients with MS. Longitudinal studies are needed to determine the potential role that these imaging metrics could play as outcome measures or in aiding patient selection for clinical trials of remyelinating therapies.

Disclosures: Fang Yu—RELATED: Grant: Radiological Society of North America Research Resident Grant, Comments: funds to support the purchase of research-related equipment and software, such as Matlab and SPSS.* Aapo Nummenmaa-RELATED: Grant: National Institutes of Health, Comments: Work was partially supported by the National Institutes of Health under award No. R00EB015445.* Lawrence L. Wald-RELATED: Grant: National Institutes of Health*; UNRELATED: Employment: Massachusetts General Hospital. Eric C. Klawiter-RELATED: Grant: National Institutes of Health, National MS Society, Altschul Foundation, Comments: National Institutes of Health K23NS078044, NMSS PR-1412-02194*; UNRELATED: Consultancy: Acorda, Alexion Pharmaceuticals, Atlas5D, Biogen, Celgene, EMD Serono, Genentech, MedDay; Grants/Grants Pending: AbbVie, Atlas5D, Biogen, EMD Serono, Genentech, Genzyme.* Susie Y. Huang-RELATED: Grant: Conrad N. Hilton Foundation, Comments: research grant.* *Money paid to institution.

\section{REFERENCES}

1. Dutta $\mathrm{R}$, Trapp $\mathrm{BD}$. Mechanisms of neuronal dysfunction and degeneration in multiple sclerosis. Prog Neurobiol 2011;93:1-12 CrossRef Medline

2. Kremer D, Akkermann R, Kury P, et al. Current advancements in promoting remyelination in multiple sclerosis. Mult Scler 2019;25:7-14 CrossRef Medline

3. Mallik S, Samson RS, Wheeler-Kingshott CA, et al. Imaging outcomes for trials of remyelination in multiple sclerosis. J Neurol, Neurosurg, Psychiatry 2014;85:1396-1404 CrossRef Medline

4. Jensen JH, Helpern JA, Ramani A, et al. Diffusional kurtosis imaging: the quantification of non-gaussian water diffusion by means of magnetic resonance imaging. Magn Reson Med 2005;53:1432-40 CrossRef Medline

5. Zhang H, Schneider T, Wheeler-Kingshott CA, et al. NODDI: practical in vivo neurite orientation dispersion and density imaging of the human brain. Neuroimage 2012;61:1000-16 CrossRef Medline

6. Assaf Y, Blumenfeld-Katzir T, Yovel Y, et al. AxCaliber: a method for measuring axon diameter distribution from diffusion MRI. Magn Reson Med 2008;59:1347-54 CrossRef Medline

7. Huang SY, Tobyne SM, Nummenmaa A, et al. Characterization of axonal disease in patients with multiple sclerosis using high-gradient-diffusion MR imaging. Radiology 2016:280:244-51 CrossRef Medline
8. Fu X, Shrestha S, Sun M, et al. Microstructural white matter alterations in mild cognitive impairment and Alzheimer's disease: study based on neurite orientation dispersion and density imaging (NODDI). Clin Neuroradiol 2019 June 7. [Epub ahead of print] CrossRef Medline

9. Huang SY, Nummenmaa A, Witzel T, et al. The impact of gradient strength on in vivo diffusion MRI estimates of axon diameter. Neuroimage 2015;106:464-72 CrossRef Medline

10. Veraart J, Fieremans E, Rudrapatna U, et al. Breaking the power law scaling of the dMRI signal on the Connectom scanner reveals its sensitivity to axon diameters. In: Proceedings of the Annual Meeting of the International Society for Magnetic Resonance in Medicine. Paris, France; June 16-21, 2018: 252

11. Veraart J, Fieremans E, Novikov DS. On the scaling behavior of water diffusion in human brain white matter. Neuroimage 2019;185:379-87 CrossRef Medline

12. Henkelman RM, Huang X, Xiang QS, et al. Quantitative interpretation of magnetization transfer. Magn Reson Med 1993;29:759-66 CrossRef Medline

13. MacKay A, Whittall $\mathrm{K}$, Adler $\mathrm{J}$, et al. In vivo visualization of myelin water in brain by magnetic resonance. Magn Reson Med 1994;31:673-77 CrossRef Medline

14. Mezer A, Yeatman JD, Stikov N, et al. Quantifying the local tissue volume and composition in individual brains with magnetic resonance imaging. Nat Med 2013;19:1667-72 CrossRef Medline

15. Stikov N, Campbell JS, Stroh T, et al. In vivo histology of the myelin g-ratio with magnetic resonance imaging. Neuroimage 2015;118:397405 CrossRef Medline

16. Cercignani M, Giulietti G, Dowell NG, et al. Characterizing axonal myelination within the healthy population: a tract-by-tract mapping of effects of age and gender on the fiber g-ratio. Neurobiol Aging 2017;49:109-18 CrossRef Medline

17. Hagiwara A, Hori M, Yokoyama K, et al. Analysis of white matter damage in patients with multiple sclerosis via a novel in vivo MR method for measuring myelin, axons, and g-ratio. AJNR Am J Neuroradiol 2017;38:1934-40 CrossRef Medline

18. Berman S, West KL, Does MD, et al. Evaluating g-ratio weighted changes in the corpus callosum as a function of age and sex. Neuroimage 2018;182:304-13 CrossRef Medline

19. Campbell JSW, Leppert IR, Narayanan S, et al. Promise and pitfalls of g-ratio estimation with MRI. Neuroimage 2018;182:80-96 CrossRef Medline

20. Duval T, Le Vy S, Stikov N, et al. G-ratio weighted imaging of the human spinal cord in vivo. Neuroimage 2017;145:11-23 CrossRef Medline

21. Keil B, Blau JN, Biber S, et al. A 64-channel 3T array coil for accelerated brain MRI. Magn Reson Med 2013;70:248-58 CrossRef Medline

22. Huang SY, Witzel T, Fan Q, et al. TractCaliber: axon diameter estimation across white matter tracts in the in vivo human brain using $300 \mathrm{mT} / \mathrm{m}$ gradients. In: Proceedings of the Annual Meeting of the International Society for Magnetic Resonance in Medicine. Toronto, Ontario, Canada; May 20 to June 5, 2015

23. Fan Q, Witzel T, Nummenmaa A, et al. MGH-USC Human Connectome Project datasets with ultra-high b-value diffusion MRI. Neuroimage 2016;124:1108-14 CrossRef Medline

24. Andersson JL, Skare S, Ashburner J. How to correct susceptibility distortions in spin-echo echo-planar images: application to diffusion tensor imaging. Neuroimage 2003;20:870-88 CrossRef Medline

25. Smith SM, Jenkinson M, Woolrich MW, et al. Advances in functional and structural MR image analysis and implementation as FSL. Neuroimage 2004;23(Suppl 10):S208-19 Medline

26. Andersson JLR, Sotiropoulos SN. An integrated approach to correction for off-resonance effects and subject movement in diffusion MR imaging. Neuroimage 2016;125:1063-78 CrossRef Medline

27. Fan Q, Nummenmaa A, Witzel T, et al. Axon diameter mapping independent of crossing structures using spherical mean technique. 
In: Proceedings of the Annual Meeting of the International Society for Magnetic Resonance in Medicine. Paris, France; June 16-21, 2018

28. Huang SY, Tobyne SM, Nummenmaa A, et al. Characterization of axonal pathology independent of fiber crossings in multiple sclerosis using high-gradient diffusion MRI. Radiology 2016;280:244-51 CrossRef Medline

29. Dale AM, Fischl B, Sereno MI. Cortical surface-based analysis, I: segmentation and surface reconstruction. Neuroimage 1999;9:17994 CrossRef Medline

30. Fischl B, van der Kouwe A, Destrieux C, et al. Automatically parcellating the human cerebral cortex. Cereb Cortex 2004;14:11-22 CrossRef Medline

31. Lindemer ER, Salat DH, Smith EE, et al. White matter signal abnormality quality differentiates mild cognitive impairment that converts to Alzheimer's disease from nonconverters. Neurobiol Aging 2015;36:2447-57 CrossRef Medline

32. Plemel JR, Liu W-Q, Yong VW. Remyelination therapies: a new direction and challenge in multiple sclerosis. Nat Rev Drug Discov 2017;16:617-34 CrossRef Medline

33. Stangel M, Kuhlmann T, Matthews PM, et al. Achievements and obstacles of remyelinating therapies in multiple sclerosis. Nat Rev Neurol 2017;13:742-54 CrossRef Medline

34. Berman S, West KL, Does MD, et al. Evaluating g-ratio weighted changes in the corpus callosum as a function of age and sex. Neuroimage 2018;182:1304-13 CrossRef Medline
35. Levesque IR, Giacomini PS, Narayanan S, et al. Quantitative magnetization transfer and myelin water imaging of the evolution of acute multiple sclerosis lesions. Magn Reson Med 2010;63:633-40 CrossRef Medline

36. Jelescu IO, Veraart J, Adisetiyo V, et al. One diffusion acquisition and different white matter models: how does microstructure change in human early development based on WMTI and NODDI? Neuroimage 2015;107:242-56 CrossRef Medline

37. Kunz N, da Silva AR, Jelescu IO. Intra- and extra-axonal axial diffusivities in the white matter: which one is faster? Neuroimage 2018;181:314-22 CrossRef Medline

38. Fan Q, Nummenmaa A, Wichtmann B, et al. Validation of diffusion MRI estimates of compartment size and volume fraction in a biomimetic brain phantom using a human MRI scanner with $300 \mathrm{mT} / \mathrm{m}$ maximum gradient strength. Neuroimage 2018;182:469-78 CrossRef Medline

39. Duval T, Saliani A, Nami H, et al. Axons morphometry in the human spinal cord. Neuroimage 2019;185:119-28 CrossRef Medline

40. Frischer JM, Weigand SD, Guo Y, et al. Clinical and pathological insights into the dynamic nature of the white matter multiple sclerosis plaque. Ann Neurol 2015;78:710-21 CrossRef Medline

41. Abelev B, Adam J, Adamova D, et al; ALICE Collaboration. A measurement of quarkonium production at forward rapidity in [Formula: see text] collisions at [Formula: see text]TeV. Eur Phys J C Part Fields 2014;74:2974 CrossRef Medline 